\title{
FACTORES CONTEXTUALES IMPLICADOS EN LA ELECCIÓN DEL SISTEMA DE INFORMACIÓN INTERNO*
}

\author{
ANA C. URQUIDI MARTIN"**, CARMEN TAMARIT AZNAR ${ }^{* * * *}$ \& VICENTE M. RIPOLL FELIU ${ }^{* * * * *}$ \\ UNIVERSITAT DE VALĖNCIA (ESPAÑA)
}

Recibido/ Received/ Recebido: 08/12/14 - Aceptado/ Accepted / Aprovado: 26/10/15

\begin{abstract}
Resumen
El objetivo del presente trabajo es identificar los factores contextuales que potencialmente influyen o están implicados en la elección y eficiencia del sistema de información interno. La investigación planteada obedece a un estudio explicativo. Se centra en un solo sector económico, debido a que permite acotar los efectos derivados de las condiciones de mercado, del ciclo económico y de la tecnología de producción. El sector elegido ha sido el hotelero. Se concluye que el rendimiento de una organización depende del acoplamiento entre la estrategia, la estructura y el sistema de información interno implantado en la organización.

Palabras clave: Sistema de información interno; Elección; Eficiencia; Organización.
\end{abstract}

\section{CONTEXTUAL FACTORS INVOLVED IN THE ELECTION OF AN INTERNAL INFORMATION SYSTEM}

\begin{abstract}
The aim of this study is to identify the contextual factors that potentially influence or are involved in the choice and efficiency of the internal information system. The raised research follows an explanatory study. It focuses on a single economic sector, because it allows limiting the effects of market conditions, the economic cycle and production technology. The chosen sector has been the hotel
\end{abstract}

\footnotetext{
Artículo de investigación científica.

** Doctora en Ciencias Económicas y Empresariales por la Universidad de Valencia. Miembro del Equipo Valenciano de Investigación en Gestión Estratégica de Costes (IMACCev), y de la Asociación Iberoamericana de Control de Gestión AICOgestión. Profesora del Departamento de Contabilidad de la Universitat de València. Directora del Master "Economía de la Salud y Gestión de la Calidad Sanitaria". Dirección postal: Av. de Blasco Ibáñez, 13, 46010 València, Valencia (España). Teléfono: +34 963864100 . Correo electrónico: ana.c.urquidi@uv.es.

*** Doctora en Ciencias Económicas y Empresariales por la Universidad de Valencia. Profesora Titular del Departamento de Contabilidad de la Universitat de València. Directora del Master "Análisis, Evaluación y Estrategias de actuación para maximizar el beneficio". Miembro del Equipo Valenciano de Investigación en Gestión Estratégica de Costes (IMACCev), de la Asociación Iberoamericana de Control de Gestión. Correo electrónico: Carmen.tamarit@uv.es.

***** Doctor en Ciencias Económicas y Empresariales. Presidente de la Asociación Iberoamericana de Control de Gestión. Director del Master de Finanzas y Control de Gestión de la Universidad de Valencia (premio Universidad-Sociedad en la edición XVI del Consejo Social de la Universidad de Valencia a la actividad formativa de postgrado y especialización) y del Máster en SAP ERP Controlling y Business Intelligence. Director del grupo de investigación IMACCev (equipo valenciano de investigación en gestión estratégica de costes). Miembro del Grupo de Reflexión Estratégica para el impulso de la cooperación Universidad Empresa. Director de la línea de investigación "aportaciones del management accounting a la gestión estratégica de costes. Profesor del Departamento de contabilidad de la Universitat de València. Correo electrónico: vicente.ripoll@uv.es.
} 
industry. It is concluded that the performance of an organization depends on the link between strategy, structure and internal reporting system implemented in the organization.

Keywords: Internal information system; Choice; Efficiency; Organization.

\title{
FATORES CONTEXTUAIS IMPLICADOS NA ESCOLHA DO SISTEMA INTERNO DE INFORMAÇÃO
}

\begin{abstract}
Resumo
O objetivo do presente trabalho é identificar os fatores contextuais que potencialmente influenciam ou estão implicados na escolha e eficiência do sistema interno de informação. A pesquisa proposta obedece a um estudo explicativo. Centra-se em um único setor econômico, devido a que com isto permite melhor dimensionar os efeitos derivados das condições de mercado, do ciclo econômico e da tecnologia de produção. O setor escolhido foi o hoteleiro. Conclui-se que o rendimento de uma organização depende do acoplamento entre a estratégia, a estrutura e o sistema interno de informação implantado na organização.
\end{abstract}

Palavras chave: Sistema interno de informação; Escolha; Eficiência; Organização.

Urquidi, A., Tamarit, C. \& Ripoll, V. (2016). Factores contextuales implicados en la elección del sistema de información interno. En: Revista de la Facultad de Ciencias Económicas de la Universidad Militar Nueva Granada. rev.fac.cienc.econ, XXIV No1, DOI: http://dx.doi.org/10.18359/rfce.1621.

JEL: $M 15, M 41, M 49$.

\section{Introducción}

Es un hecho reconocido que durante las últimas décadas se viene produciendo una vertiginosa transformación de la economía mundial. Por un lado gracias a las innovaciones tecnológicas, el proceso productivo cambia, desaparece la producción continua, se buscan sistemas de producción más flexibles, con líneas de productos poco repetitivas, acortando el ciclo de vida de éstos, y adaptándose continuamente a las necesidades de los clientes, logrando además, reducciones en las mermas, en la subactividad y en la producción en curso.

Por otro lado, la globalización o mundialización ha ampliado las oportunidades para que economías individuales participen más activamente de las redes internacionales de producción, ya que ahora las fronteras nacionales son permeables, y este nuevo contexto facilita la creación de diferentes formas de organización y conexión económica y social a nivel mundial (Koen, 2004), volviendo anacrónicos cualquier aislacionismo.
Por lo que las organizaciones de todos los sectores económicos han de enfrentarse a mayores niveles de competencia y cambio, lo que implica, más posibilidades de negocio, pero con niveles notables de incertidumbre ante el futuro. Así pues, el reto para la empresa es crear nuevas ventajas competitivas, ya que el éxito, no sólo está ligado a la minimización de los costes, sino también a la satisfacción del cliente, la innovación, la calidad, y la adaptabilidad del producto o del servicio (Bisbe \& Otley, 2004; Kanter, 2001; Ylinen \& Gullkvist, 2014).

Estas tendencias, obligan a las organizaciones a redefinir los fundamentos de su negocio y buscar soluciones que les permitan sobrevivir y prosperar. La información juega un papel primordial en este escenario (Siti-Nabiha \& Scapens, 2005), constituyéndose en el pilar básico de una sociedad cada vez más interconectada y globalizada. Además de convertirse en un flujo vital que precisan las organizaciones para funcionar de forma eficiente y competitiva en un escenario siempre cambiante (Mahlendorf et al., 2014). 
El acceso y gestión de dicha información requiere del diseño y montaje de una infraestructura organizativa y tecnológica que responda a las necesidades de la empresa y, que al mismo tiempo, esté en sintonía con la estrategia y la cultura corporativa. Es decir, se hace necesario construir un conjunto integrado de sistemas de información, Olaru et al. (2014), el cual ha de estar implantado en una plataforma adecuada, de forma que permita el funcionamiento fluido de las diversas áreas del negocio (Chenhall et al., 2013).

Por todo lo anterior, se aborda el presente trabajo, con el objetivo de identificar los factores contextuales que potencialmente influyen o están implicados en la elección y eficiencia del sistema de información interno.

Estos factores se han agrupado en función de la influencia o control que pueda ejercer la organización sobre ellos. Así pues, están los factores exógenos o no controlables por parte de la organización, como el entorno (Baines \& Langfield-Smith, 2003; Burns \& Stalker, 1961), y los factores endógenos o controlables por parte de la organización entre los que destacan la estrategia (Langfield-Smith, 1997; Porter, 1985), y la estructura (Drazin \& Van de Ven, 1985; Lawrence \& Lorch, 1967).

La investigación planteada obedece a un estudio explicativo que intenta avanzar en el conocimiento de los factores determinantes en la elección y eficiencia del sistema de información interno. Así pues, el punto de partida es una revisión de las diferentes aportaciones realizadas en el ámbito de estudio. Esta revisión permite plantear las hipótesis a contrastar. Las cuales con posterioridad son verificadas, en la investigación de campo llevada a cabo, y finalmente, se discuten los resultados empíricos en base a la revisión teórica realizada, cerrando el trabajo, con la presentación de las conclusiones.

La investigación se ha centrado en un solo sector económico, ya que este hecho permite acotar los efectos derivados de las condiciones de mercado, del ciclo económico y de la tecnología de producción. El sector elegido ha sido el hotelero, debido al papel cada vez más relevante que ocupa en las modernas economías. La importancia del sector se generaliza a nivel mundial, las tendencias del mercado indican un crecimiento sostenido en las cuentas de llegadas de turistas e ingresos y los instrumentos estadísticos diseñados para medir el comportamiento de esta actividad señalan lo que parece ser una firme recuperación en todo el mundo.

\section{Marco teórico}

La necesidad de un sistema de información interno en las organizaciones para su buen gobierno y funcionamiento ha sido ampliamente analizada por diferentes autores que han investigado sobre este tema (Laitinen 2014). Pero si se repasa cual es el sistema más eficiente, se observa que no existe un sistema de información interno aplicable a todas las organizaciones y en cualquier situación, ya que dependerá de las circunstancias o factores contextuales en las que se encuentre (Bastian \& Muchlish, 2012).

Para que el sistema de información sea eficiente se necesita la alineación entre varios factores organizativos dentro de una situación empresarial determinada (Donalson, 2001; Lawrence \& Lorsch, 1967; Naranjo-Gil \& Alvarez-Dardet, 2006; Zhanga et al., 2012). Todos los componentes de la organización deben ajustarse entre si para que la organización mejore su rendimiento. A continuación se aborda sucintamente los factores que se analizan en el trabajo, resumiendo sus principales características.

\subsection{Entorno}

El entorno es todo lo que se encuentra desde los límites de la organización hacia fuera y donde los gerentes no poseen capacidad de influencia o control. Dado lo cual, se puede afirmar, que conocer el entorno donde desarrolla una organización su actividad es un factor determinante a la hora de elegir el sistema de información contable (Chenhall \& Morris, 1986).

Para Emmanuel et al., (1991) dentro del entorno, se pueden incluir cuatro variables:

- Incertidumbre: que mide el nivel de predicción que sobre el entorno se puede realizar en la organización. 
- Nivel de competencia que tiene la empresa en precios, productos, tecnología, etc.

- Heterogeneidad: que viene determinada por el número de productos o mercados diferentes en que se desenvuelve la actividad de la organización.

- Apoyo por parte de las instituciones públicas o privadas.

\subsection{Estrategia}

La estrategia es el patrón o plan que integra las principales metas y políticas de una organización, a partir de la cual, se establece una secuencia coherente de acciones a realizar. Así pues, la estrategia engloba tanto los fines como los medios, y consiste en un flujo de decisiones conscientemente planeadas por la dirección, que afectan a los diversos niveles jerárquicos y a las diferentes áreas de actividad de la organización.

Las investigaciones han demostrado que la estrategia de una organización es una respuesta a su entorno (Baines \& Langfield-Smith, 2003; González-Benito 2010). Por lo que ante diferentes entornos a las organizaciones les interesarán diferentes estrategias. Tres son las opciones estratégicas propuestas por Porter (1985): el liderazgo en costes, la diferenciación y el enfoque.

En una estrategia de liderazgo en costes la empresa se propone ser el productor de menor coste en su sector industrial. Para conseguir este objetivo la empresa ha de ser eficiente, tener unos gastos generales bajos, con intolerancia al desperdicio, y muchos elementos de control.

Por otro lado, si lo que busca es una estrategia de diferenciación, la empresa le creará a su producto o servicio algo que sea percibido en toda la industria como único, y dado que el mercado entiende que el producto es diferente y está adaptado a sus propias necesidades están dispuestos a pagar un precio superior.

Si lo que va a implantar es una estrategia de enfoque, la empresa ha de elegir un panorama de competencia estrecho dentro de un sector industrial. El "enfocador" selecciona un grupo o segmento del sector industrial donde realiza su actividad y ajusta su estrategia a servirlos con la exclusión de otros, logrando de este modo una ventaja competitiva general.

\subsection{Estructura}

Por estructura organizativa se entiende "la suma total de las formas en las que una organización divide sus recursos humanos en tareas diferentes y su posterior coordinación" (Hodge et al., 2003). Esta definición reconoce dos elementos claves, la diferenciación y la integración.

Así pues, Lawrence \& Lorch (1967) se refieren a estructura, genéricamente, como a la manera en que la organización es diferenciada e integrada. La diferenciación lo que mide es la distribución del poder dentro de la organización, la variedad de los grupos y su participación en las decisiones. La integración es definida como el grado en el que las actuaciones de las diferentes subunidades son compatibles con el objetivo global de la organización. Los mecanismos para lograr la diferenciación implican descentralización de la autoridad, mientras que la integración implica reglas, procedimientos formales y políticas comunes.

En el ámbito estructural, la clasificación más conocida es la que agrupa a las empresas en función de su grado de formalización, obteniendo dos tipos de estructuras: orgánicas o mecánicas. Las características básicas de estas organizaciones fueron definidas por Burns \& Stalker (1961).

Así, las estructuras orgánicas se caracterizan por la necesidad de personas con conocimientos específicos y experiencia para la realización de la tarea común. En este caso se produce un continuo ajuste y continua redefinición de las tareas individuales, a través, de la interacción con otros miembros. Por ello, existe un núcleo informal de control, autoridad y comunicación. También se da una mayor frecuencia de comunicaciones horizontales, en vez de verticales, y el contenido de la comunicación consiste en información y consejo, en lugar de instrucciones y decisiones como ocurre en las estructuras mecánicas. 
Por otra parte, las estructuras mecánicas se caracterizan por la diferenciación especializada de sus funciones, es decir, que cada tarea se realiza con técnicas y propósitos propios, que no tienen que coincidir con los del resto de las tareas. Este tipo de estructuras se denota por el uso de la jerarquía como mecanismo de coordinación y por un núcleo de control, con autoridad y comunicación formal.

\subsection{Sistema de información interno}

La información relevante es aquella que aumenta el conocimiento y reduce la incertidumbre respecto al problema que se va a considerar. Así pues, la información se convierte en un recurso esencial y estratégico, que se podrá obtener por varias fuentes. Al definir la contabilidad como una ciencia de naturaleza económica y empírica, cuyo objeto material es la realidad económica y social de la empresa, tanto en su aspecto cualitativo como cuantitativo, presente, pasado y futuro, cuyo objeto formal es la captación, representación, medida y comunicación de esa realidad; siendo su objetivo, aportar datos útiles para la toma de decisiones, se considera ésta como el sistema de información cuantitativa y cualitativa principal de la empresa (Johnson \& Kaplan 1987).

De ahí la importancia a la hora de diseñar el sistema de información. Cuando se analizan los cambios acaecidos en los procesos productivos, acompañados de importantes modificaciones en los sistemas de organización de las empresas, se observa que se están produciendo modificaciones importantes en la estructura de los costes. El aumento de la mecanización e informatización de los procesos, por ejemplo, ha dado lugar a que el coste del personal en muchas empresas pase a ser un mero elemento de control o apoyo a los equipos, que son los que desarrollan realmente las tareas productivas, pasando así a tener una importancia ínfima en relación con los costes totales de la actividad productiva.

Todo lo anterior ha producido transformaciones en los sistemas internos de producción y de control, y consecuentemente, en los sistemas de gestión, los cuales han dejado de plantearse como único elemento de competitividad, la minimización de los costes, de tal manera que los productos buscan ob- jetivos cualitativos tales como: la calidad, los tiempos de respuesta, etc. (Ripoll \& Urquidi, 2010).

Para hacer frente a estas necesidades surgen las denominadas Nuevas Técnicas de Gestión. Uno de los problemas que se encuentra en este apartado es semántico, ya que al hacer un repaso de la literatura se observa que los autores utilizan diferentes términos para los mismos conceptos, así utilizan la denominación prácticas contables, autores como Abdel-Maksoud (2004); Chenhall \& Langfield-Smith (1998a, 1998b) y Guilding et al. (2000), técnicas contables autores como Bright et al. (1992) y Cooper (1996) o herramientas contables o de control denominación utilizada por Puolamäki (2004) y Rigby (2003), entre otros.

Independientemente de la denominación que se utilice todas estas nuevas técnicas, prácticas o herramientas, surgen como consecuencia de las limitaciones de los sistemas tradicionales para hacer frente a los cambios que se han producido en el entorno (Rigby, 2003).

Aunque el número de nuevas técnicas de gestión es más amplio, se ha seleccionado las que se han considerado más utilizadas por el sector estudiado (Mauri, 2012; Rigby \& Bilodeau, 2013), y estas son: planificación estratégica; benchmarking; C.R.M. customer relation management; outsourcing; segmentación de clientes; cuadro de mando; TQ Management; ABC/ ABM; y gestión del conocimiento.

\section{Hipótesis}

\subsection{Entorno}

Un entorno se puede considerar hostil, ya sea por la excesiva competencia, por la heterogeneidad de productos o por la incertidumbre que perciben los gerentes para predecir la evolución de éste. Así pues, la información que aporta la contabilidad de gestión se va a convertir en un factor clave para asegurar que el desempeño, de la empresa dentro del entorno en el que interactúa, sea eficiente. Trabajos como los de Chenhall \& Morris (1986), Gordon \& Miller (1976) y McManus (2013) observan una relación positiva entre la incertidumbre ambiental y necesidades de información oportuna, actual. 
La estrategia de una organización debe ser una respuesta al entorno en que se desenvuelve, y ha de existir una correspondencia apropiada entre la estrategia y el entorno, ya que ésta, puede ser clave en el éxito de una organización (Baines \& LangfieldSmith, 2003). La relación entre ambas variables ha sido evidenciada en estudios empíricos, tales como los de Fuchs et al. (2000) y Garrigos-Simon \& Narangajavana (2005), los cuales observan que las empresas que alinean los elementos de la estrategia con el entorno tienen más éxito; Chong \& Chong (1997) encuentra una asociación positiva entre la incertidumbre percibida y la estrategia; Perea et al. (1997) y Sim \& Killough (1998), en sus trabajos empíricos, demuestran que cuando se produce un aumento de la competencia y ésta responde a las exigencias de los clientes, de forma cada vez más sofisticada, la organización está poniendo énfasis en una estrategia de diferenciación, Becerra et al. (2013), que acentúa los aspectos relacionados con la orientación hacia el cliente, entre los que destacan la calidad, la flexibilidad y una constante innovación en los productos.

De aquí que se plantee la primera hipótesis de la siguiente manera:

H1. El aumento de la oferta hotelera influye significativamente en la utilización de una estrategia de diferenciación.

La literatura sobre diseño organizativo propone que las organizaciones que soportan mucha presión externa, tienden a hacer cambios en su estructuras a corto plazo mediante la adopción de controles orgánicos (Khandwalla, 1977). De manera más concreta, se ha observado en trabajos empíricos, que ante cambios en el entorno las organizaciones pueden responder con una reorganización de sus procesos productivos, y con la adopción de nuevas estructuras organizativas (Keidel, 1994; Parthasarty \& Sethi, 1993).

De este modo, la adopción de estas estructuras organizativas está asociada con la descentralización y con una disminución en la formalización de las tareas a realizar (Chenhall \& Langfield-Smith, 1998a; ClaverCortés et al., 2007), ya que lo que se pretende, es asegurar respuestas rápidas $e$ innovadoras en ambientes complejos y dinámicos.
De igual manera, las conclusiones que obtienen Kajüter \& Kulmala (2005) apuntan en este sentido, así pues, en ambientes competitivos las organizaciones necesitan recortar costes y para ello crean equipos de trabajos multifuncionales, que eliminan las continuas interacciones entre subunidades, influyendo de manera significativa en el éxito de la organización. A su vez, Chapman (1998) propone flexibilidad a la hora de planificar en contabilidad, cuando exista incertidumbre. A tenor de los resultados obtenidos en su estudio, concluye que ésta es la mejor manera de enfrentarse a todos los cambios imprevisibles que se presenten.

Esto nos lleva a formular la segunda hipótesis en los siguientes términos:

H2. El aumento de los niveles de competencia influye significativamente en la utilización de una estructura orgánica.

\subsection{Estrategia}

En función de la estrategia que elija la organización le interesará una estructura organizativa diferente (Govindarajan, 1984; Miller, 1988). Así, las organizaciones que adopten una estrategia de diferenciación, Brenes et al. (2014), tienden a afrontar un grado más alto de incertidumbre. El motivo principal es que éstas obtienen su ventaja competitiva adaptándose a las necesidades de sus clientes ante cualquier cambio de la demanda, siempre con la máxima calidad. Para lo cual, dichas organizaciones deben tener una producción flexible y un rediseño continuo del producto (Jermias \& Gani, 2004). Todo lo anterior, condiciona a la estructura, debido principalmente, a que la descentralización y la baja formalización, fomenta la participación de los empleados y el compromiso que adquieren es una baza fundamental para el éxito de la organización con este tipo de estrategia (Banker et al., 1993). Estudios como los de Baines \& Langfield-Smith (2003); Burns \& Stalker (1961); Cugueró-Escofet \& Rosanas (2013); Govindarajan (1984); Jermias \& Gani (2004); Lawrence \& Lorsch (1967); Sanchez Quiros (2003); Smith et al. (2002); Zhenga et al. (2010), han observado que la estructura es una variable decisiva en función de la estrategia que implante la organización. 
De ahí que se formule la hipótesis 3 en los siguientes términos:

H3. La utilización de una estrategia de diferenciación influye significativamente en la utilización de estructuras orgánicas.

\subsection{Sistemas de información internos}

La estructura de la organización, Van der Voet (2014), es una variable determinante de la elección del sistema de información contable. Debido principalmente, a la influencia que tiene en la eficiencia de la organización (Gerdin, 2005). Cuando hay cambios en los sistemas de información, la estructura que tenga la organización será determinante para el fomento o el rechazo del nuevo sistema (Hodge et al., 2003).

La relación entre la estructura de la organización y los sistemas de información internos, ha sido analizada a partir de la taxonomía de estructuras mecánicas y orgánicas propuestas por Burns \& Stalker (1961). En la literatura, las organizaciones que presentan una estructura mecánica han sido asociadas con tradicionales sistemas de información contables (Gerdin, 2005; Kaplan, 1984; Macintosh, 1994). Por otro lado, estudios empíricos encuentran una relación positiva entre la adopción de las nuevas técnicas de contabilidad de gestión y la estructura organizativa. Así pues, las organizaciones con una estructura orgánica tendrán una mayor facilidad para adoptar innovaciones técnicas (Chenhall \& Morris, 1995, Chenhall, 2003; Henri 2006, Fullerton \& McWatter, 2002; Jørgensen \& Messner, 2009; Mundy, 2010; Ylinen \& Gullkvist, (2014).

Lo expuesto anteriormente nos conduce a enunciar la hipótesis 4 de la siguiente manera:
H4. La adopción de una estructura orgánica, influye significativamente en la utilización de nuevas técnicas de contabilidad de gestión.

\subsection{Rendimiento de la organización}

Se parte de la premisa de la inexistencia de una única y mejor forma de gestionar y configurar las organizaciones, planteando la necesidad de una coordinación entre la estrategia (Green et al., 2008), la estructura organizativa, el sistema de control de gestión y el contexto donde opera dicha organización (Chenhall, 2003).

Thomas \& Ramaswamy (1996) se apoyaron en el concepto de ajuste para afirmar que debe existir una congruencia entre los estilos de gestión (Werbel \& DeMarie 2005), y la orientación estratégica de la organización. La mayoría de los estudios se han centrado en analizar esta relación, bajo esta perspectiva, autores como Abernethy \& Bouwens (2005), Christensen \& Demski (2003), Hunt \& Morgan (1995), Shields et al. (2000), Sinkula et al. (1997), y Tuominen \& Möller (1996), consideran que una estrategia orientada al mercado y una estructura organizativa orgánica permite la obtención de un rendimiento superior.

De acuerdo con lo anterior se formula la siguiente hipótesis:

H5 La utilización de nuevas técnicas de contabilidad de gestión está significativamente relacionada con una mejora en el rendimiento.

Un resumen de todas las hipótesis planteadas aparece expuesto en la figura 1.

Figura 1. Modelo teórico

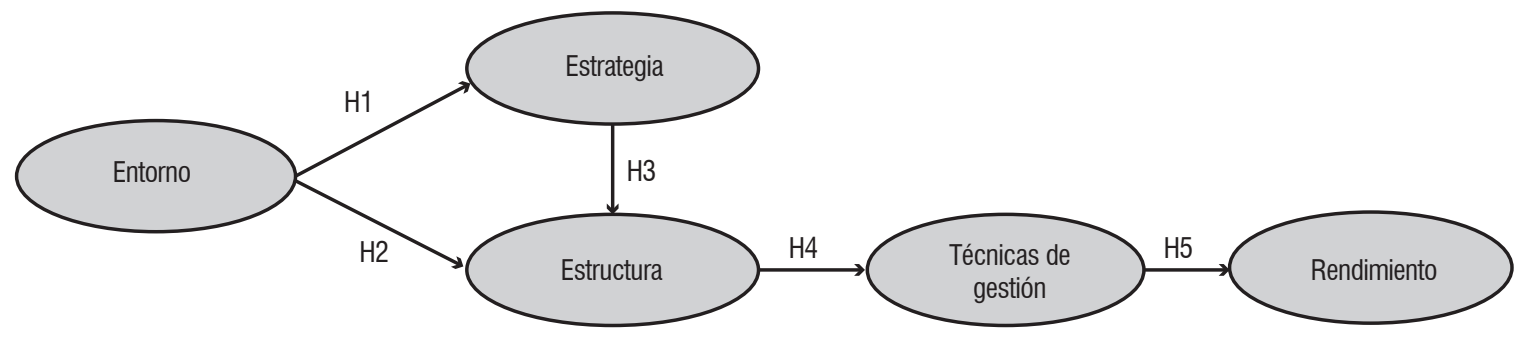

Fuente: Elaboración propia. 


\section{Estudio empírico}

\subsection{Recogida de los datos}

La investigación se centra en el sector hotelero español. Los motivos que nos han conducido a esta selección son varios. En primer lugar, hay un creciente interés por el sector servicios que se recoge en la literatura en los últimos años, debido principalmente, al rápido crecimiento que la actividad de dicho sector ha experimentado en las últimas décadas, en comparación con el sector manufacturero (Cuadrado-Roura \& Maroto-Sánchez, 2012).

Adicionalmente, el sector servicios en nuestro país, es el más importante en términos de PIB y de creación de empleos. Y al analizar la cuenta servicios en la Balanza de Pagos, el subsector turismo, es una de las partidas más importantes de ingresos, representando el $10.9 \%$ del PIB y generando el $12 \%$ del empleo en el país ya que en 2012 llegaron a España 57,7 millones de turistas internacionales, y el total de pernoctaciones en hoteles ascendió a 281,4 millones (IET, 2012). Pero, a pesar del incremento en el número de turistas, el Índice de Tendencia de Competitividad Turística (ITCT), que se elabora a partir de la evolución de los precios de la oferta española en relación a otros países y la variación relativa de los tipos de cambio nominales que encontrará el turista cuando llegue a España, en el año 2011, refleja cierta pérdida de competitividad vía precios, con respecto al año anterior, por lo que existe un interés, en mejorar la productividad de un sistema operativo con una estructura de costes de producción poco eficiente. Eso a pesar de los buenos datos que se han dado del ejercicio 2013.

En el VII Foro del Liderazgo Turístico, se ha puesto de manifiesto que el número de turistas que visitaron España durante el año 2013 ascendió a 60,6 millones, lo que supone un 5,6\% más respecto al ejercicio anterior y representa un récord histórico. España ha recuperado la tercera posición de país receptor de turistas, al superar a China y situarse por detrás de Francia (83 millones) y Estados Unidos (67 millones). España está en camino desde el punto de vista de la balanza de pagos, de superar por vez primera los 45.000 millones de euros, un dato que ha explicado que es también el mejor de la serie histórica.
Además, se ha optado por realizar un estudio empírico unisectorial: establecimientos hoteleros de cuatro $y$ cinco estrellas, que prestan sus servicios en territorio español, independientemente de la elección modal que presente la organización. La muestra de empresas se determinó a partir del directorio "Listado de establecimientos turísticos" de la página web oficial de Turismo de España ${ }^{1}$.

Esta delimitación de la población ha dado como resultado una muestra de 1.762 establecimientos hoteleros, de los cuales nos contestaron 244 , esto supone una tasa de respuesta del 13,84\% del número total de empresas invitadas a participar en la tasa de respuesta del estudio.

\subsection{Medidas}

Para validar los constructos de cada uno de los factores se realizó un análisis factorial confirmatorio. Los valores del índice de modificación del modelo inicial indicaron que varios elementos con cargas de doble cruz deberían ser eliminados. Una vez eliminados, el cuestionario recogía 20 ítems organizados en bloques:

- Datos generales de clasificación del establecimiento hotelero (2 ítems).

- Conocimiento del entorno específico (nivel de predicción, nivel de competencia, heterogeneidad de la demanda y apoyo por parte de las instituciones públicas o privadas 4 ítems).

- Conocer el perfil y las características básicas de las empresas encuestadas (3 ítems).

- Conocer cuál es la estrategia seguida por la empresa (3 ítems).

- Conocer qué sistemas de información internos utilizan las empresas (4 ítems).

- Conocer el desempeño organizativo (4 ítems).

El cuestionario adopta para la mayoría de las preguntas el formato de las escala tipo Likert, con cinco

http://www.spain.info/es/. 
opciones que van desde muy en desacuerdo hasta muy de acuerdo, o desde nunca o casi nunca a siempre o casi siempre, según las preguntas.

Una vez elaborado, el cuestionario, fue revisado, en primer lugar, por un grupo de profesores doctores del Departamento Contabilidad de varias Universidades, expertos en la materia analizada y/o en cuestiones estadísticas, así como por tres gerentes de hoteles con más de 10 años de experiencia en el sector. Tras haber estudiado el cuestionario, los expertos evaluaron la relevancia que cada uno de los ítems tenía para el fenómeno que se pretendía medir, así como, la claridad y la precisión de éstos, modificándose aquellos que pudieran dar problemas a la hora de medición o interpretación respecto al concepto teórico, y los que se consideraron inicialmente como confusos, redundantes o ambiguos.

Seguidamente, se incorporaron las propuestas realizadas por los expertos y se llevó a cabo un pre-test a responsables de dos empresas pertenecientes a la población seleccionada. Se les envió el cuestionario en las mismas condiciones que lo recibirían el resto de las empresas a investigar. En términos generales, el cuestionario fue valorado positivamente y tan solo se hicieron ligeras modificaciones que se incorporaron a la versión definitiva.

\subsection{Análisis de los datos}

La mayoría de los investigadores están de acuerdo en que la varianza común del método (la varianza que es debida al método de medición más que a los constructos que representa la medida) es un problema potencial para la investigación conductual. El error en la medida puede invalidar las conclusiones (Podsakoff \& Organ, 1986).

Para evitar esto, se han utilizado dos técnicas diferenciadas y así controlar la varianza del método común, por un lado, se ha controlado los métodos procedimentales, es decir, en el cuestionario se introduce una separación entre las variables dependientes y las variables independientes, de tal forma que el encuestado no pueda utilizar sus respuestas previas en contestar las siguientes, y así eliminar la posibilidad de que establezca relaciones de causa-efecto entre dichas variables. Además, se indica al principio de la encuesta que las respuestas son anónimas y que no existe una respuesta óptima, siendo clave para la investigación la honestidad en sus respuestas.

Por otro lado se han realizado un análisis estadístico que permiten determinar la posible presencia de la varianza del método común en la investigación. Así pues, se ha realizado el test de un único factor de Harman, el cual se realiza incluyendo todos los ítems en un análisis factorial de componentes principales. Los resultados del análisis factorial no indican la presencia de una cantidad sustancial de varianza del método común dado que no emerge un factor general que aglutine la mayoría de la varianza, dicho factor representa un $25 \%$ de la varianza, lo que indicaría que se puede afirmar que el sesgo del método común, no contamina los resultados.

Para el análisis de datos se ha utilizado el paquete estadístico SPSS 17.0 y el programa AMOS 18.0, software con el cual se correlacionaron y describieron las variables; esta técnica de análisis permite la estimación de ecuaciones de regresión múltiple de forma simultánea, en un marco único. En particular, todas las relaciones directas e indirectas en el modelo se estiman simultáneamente y, por tanto, el método permite que todas las interrelaciones entre las variables que se evalúen estén en el mismo contexto.

Se ha aplicado un modelo de ecuaciones estructurales, debido a la riqueza de este, Burkert et al., (2014), el cual, nos permite la conjunción de dos perspectivas: la psicométrica y la econométrica. La primera aborda conceptos como variables latentes (no observadas) inferidas indirectamente a partir de múltiples medidas observadas (indicadores o variables manifiestas). La segunda, la econométrica, está centrada en la predicción a partir de los modelos de análisis con variables latentes (Chin, 1998).

Así pues, estos métodos permiten combinar y confrontar la teoría con datos empíricos, y a partir de estas, ofrecer una explicación científica que vaya, Weißenberger \& Angelkort (2011), más allá de la asociación o descripción empírica. Concretamente, admite que el investigador evalúe relaciones de causalidad entre múltiples constructos 
dependientes e independientes, valore el modelo de medida a partir del conocimiento de las cargas de los ítems observados en las variables latentes o constructos, y realice análisis factoriales confirmatorios a la vez que se testan hipótesis (Chin, 1998; Gefen et al., 2000).

Los resultados del análisis de fiabilidad y de las correlaciones se recogen en la tabla 1, la cual muestra que el entorno esta positivamente correlacionado con la estrategia, con la estructura, con las denominadas nuevas técnicas de gestión, y gracias a esta correlación mejora el rendimiento de la empresa. Se aplicó la prueba de bondad de ajustes dando como índice (GFI) = 0,85, o el índice de bondad del ajuste corregido (AGFI) $=0,82$, se observa que están un poco bajos, pero se realizaron nuevos análisis como el del error de aproximación cuadrático medio (RMSEA) 0,058, un $\chi 2 / \mathrm{gl}$ de 1,82 , y el indice de ajuste comparativo (IFI) $=0,982$ gracias a los cuales se puede concluir que el modelo es satisfactorio.

Figura 2.Coeficientes de regresión del modelo propuesto

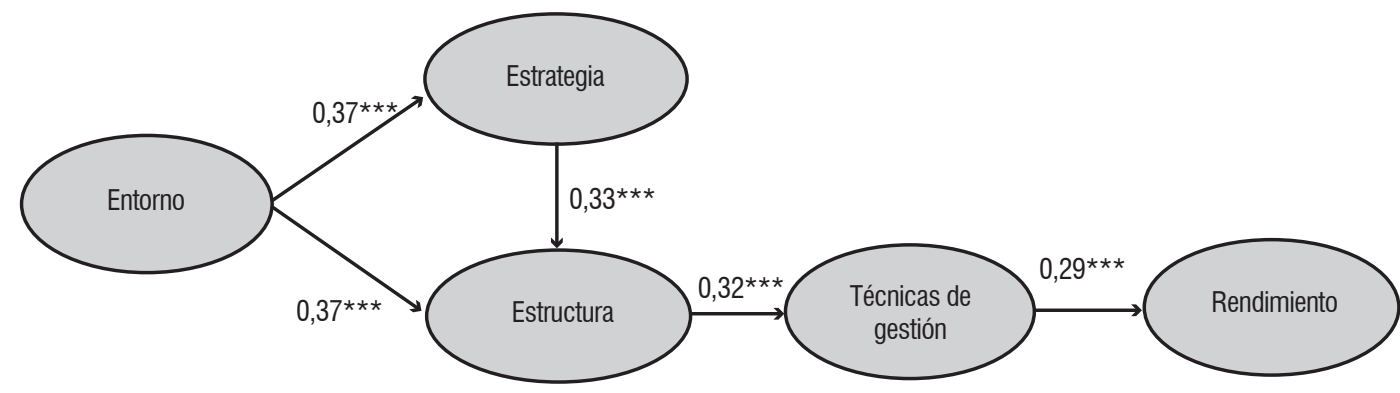

Fuente: Elaboración propia.

En esta figura 2, la evidencia empírica pone de manifiesto que la estrategia de una organización es una respuesta al entorno en que se desenvuelve, $y$ ante aumentos de la competencia, la organización tiende a responder a las exigencias de los clientes, de forma cada vez más sofisticada, destacando la calidad, la flexibilidad y una constante innovación en los productos. Por lo que se puede afirmar que el aumento de la oferta hotelera influye significativamente en la utilización de una estrategia de diferenciación.
Tabla 1. Fiabilidad, media, desviación típica y correlaciones $\left({ }^{* *} p<0,001\right)$

\begin{tabular}{|c|c|c|c|c|c|c|c|}
\hline & Mean & SD & 1 & 2 & 3 & 4 & 5 \\
\hline $\begin{array}{l}\text { Fiabilidad (alfa } \\
\text { de Cronbrach) }\end{array}$ & & & 0,93 & 0,884 & 0,901 & 0,816 & 0,793 \\
\hline 1 Entorno & 4,36 & 0,87 & & & & & \\
\hline 2 Estrategia & 5,12 & 0,81 & $0,29^{\star \star}$ & & & & \\
\hline 3 Estructura & 4,34 & 0,92 & $0,52^{* \star *}$ & $0,31^{* * *}$ & & & \\
\hline 4 Téc. Gestión & 4,52 & 0,93 & $0,42^{\star \star \star *}$ & $0,37^{\text {***}}$ & $0,32^{\star * *}$ & $0,79^{\text {*** }}$ & \\
\hline 5 Rendimientos & 3,80 & 0,94 & $0,51^{\star \star \star}$ & $0,27^{\star \star \star}$ & $0,35^{\star \star \star}$ & $0,53^{\star * *}$ & $0,56^{\star \star *}$ \\
\hline
\end{tabular}

Fuente: Elaboración propia.

Como se observa en la figura 2 el entono influye significativamente en la elección de la estrategia ( $\beta=$ $0,37 ; p<0,001)$ y de la estructura de la empresa ( $\beta$ $=0,37 ; p<0,001)$, y estas tres variables determinan la utilización de las diferentes técnicas de gestión ( $\beta$ $=0,32 ; p<0,001$ ), y gracias a la sincronización de todas estas variables el rendimiento de la empresa mejora $\beta=0,29 ; p<0,001$ ).
Por otro lado, ante entornos como los actuales los hoteleros españoles tienden a utilizar estructuras orgánicas ya que les permite hacer frente a los cambios que se van sucediendo constantemente de una forma rápida y efectiva. Así pues, el aumento de los niveles de competencia influye significativamente en la utilización de una estructura orgánica.

Con relación a nuestra tercera hipótesis, según la cual la utilización de una estrategia de diferenciación influye significativamente en la utilización de estruc- 
turas orgánicas, el parámetro $\beta=0,33$ ( $p<0,001$ ), indica, que la estrategia de diferenciación incide de forma muy importante en la elección de una estructura orgánica. El motivo principal es que este tipo de organización obtiene su ventaja competitiva adaptándose a las necesidades de sus clientes ante cualquier cambio de la demanda, siempre con la máxima calidad. Para lo cual, dichas organizaciones deben tener una producción flexible basada en una descentralización, con niveles de formalización pequeños, que fomenten la participación de los empleados.

En cuanto a la utilización de nuevas técnicas de gestión, indicar que las empresas utilizan técnicas como la gestión de la calidad, el cuadro de mando integral, la gestión del conocimiento y la segmentación de clientes debido a que estas técnicas facilitan la consecución de objetivos fijados a través de su estrategia. Así mismo, entre el conjunto de factores que facilitan la implantación con éxito de un sistema de información interno destaca la existencia de una comunicación informal dentro de la empresa, fundamentándose las decisiones en la relación personal entre el directivo y los subordinados, a través de un intercambio no regulado que surge del consenso entre los miembros de la organización, es decir la estructura orgánica, influye positivamente en la utilización e implantación de las nuevas técnicas de gestión.

Por último, la congruencia entre todos los elementos y variables es lo que va a permitir que las empresas mejoren sus resultados. Por lo que se puede afirmar que ante entornos turbulentos, la utilización de una estrategia de diferenciación, una estructura orgánica y nuevas técnicas de contabilidad de gestión está significativamente relacionada con una mejora en el rendimiento.

\section{Conclusiones}

Los cambios de todo tipo obligan a la empresa a adaptarse para sobrevivir, y este estudio proporciona una visión más amplia de cómo se están enfrentando algunas organizaciones al entorno cambiante y turbulento.

Ante estos nuevos escenarios las organizaciones necesitan información fiable, que les permita reaccio- nar de forma rápida y adecuada a las condiciones en constante cambio. Para ello, las empresas están modificando sus estrategias incluyendo como factores determinantes además del cálculo del coste, otros relacionados con la disminución del ciclo de fabricación, el tiempo de entrega, la reducción de inventarios, la satisfacción de las necesidades de los clientes, la gestión de la calidad, y la visión estratégica de los costes.

Todo lo anteriormente expuesto, complica la utilización de los sistemas de información tradicionales basados en estándares técnicos, que en las actuales condiciones son difíciles de calcular. Por tanto, los gestores necesitarán sistemas de información que estén en relación con los objetivos de la organización, con la estructura y con su manera de ser. Estos sistemas intentan incrementar la habilidad de las organizaciones para coordinar las acciones de sus miembros y para identificar los problemas que se pueden presentar.

Los resultados del estudio también revelaron que la eficacia de las organizaciones depende de la estructura organizacional, la cual, ha de ser adecuada para la estrategia elegida por la organización y el entorno donde ésta desarrolla su actividad. Por lo tanto, el diseño organizacional hace que los gerentes dirijan la vista en dos sentidos al mismo tiempo: hacia el interior y el exterior de su organización, y dado que las estrategias y los entornos cambian con el tiempo, la estructura ha de ser flexible, para adaptarse a los cambios.

Finalmente, se comprueba que el rendimiento de una organización depende del acoplamiento entre la estrategia, la estructura y el sistema de información interno implantado en la organización.

Estas contribuciones deben considerarse teniendo en cuenta las limitaciones de la investigación. En primer lugar, los resultados no son extrapolables, dada la especificidad del presente estudio, se ha dirigido a un único sector empresarial, y a un ámbito geográfico determinado. En segundo lugar, el uso de escalas de opinión otorga al estudio un cierto carácter subjetivo, aunque existe una larga tradición en el uso de las mismas en investigaciones similares. Los resultados 
obtenidos y las limitaciones indicadas nos animan a proponer algunas líneas futuras de investigación, como por ejemplo, extender el análisis a otros sectores empresariales y a diferentes ámbitos geográficos, $e$ intentar identificar otras variables que pudieran ejercer alguna influencia en el modelo propuesto para ayudar a entender mejor las conexiones existentes entre la estructura organizativa, la estrategia competitiva y los resultados.

\section{Referencias}

Abdel-Maksoud, A.B. (2004). Manufacturing in the UK: contemporary characteristics and performance indicators. En: Journal of Manufacturing Technology Management, 15: 155-171.

Abernethy, M.A. \& Bouwens, J. (2005). Determinants of accounting innovation implementation. En: Abacus, 41: 217-239.

Baines, A. \& Langfield-Smith, K. (2003). Antecedents to management accounting change: a structural equation approach. En: Accounting, Organizations and Society, 28 (7-8): 675-698.

Banker, R.D.; Potter, G. \& Schroeder, R.G. (1993). Reporting manufacturing performance measures to workers: an empirical study. En: Journal of Management Accounting Research, 5: 33-55.

Bastian, E. \& Muchlish, M. (2012). Perceived Environment Uncertainty, Business Strategy, Performance Measurement Systems and Organizational Performance. En: Procedia Social and Behavioral Sciences, 65 (3): 787-792.

Becerra, M.; Santaló, J. \& Silva, R. (2013). Being better vs. being different: Differentiation, competition, and pricing strategies in the Spanish hotel industry. En: Tourism Management, 34: 71-79.

Bisbe, J. \& Otley, D. (2004). The effects of the interactive use of management control systems on product innovation. En: Accounting, Organizations and Society, 29 (8): 709-737.

Brenes, E.; Montoya, D. \& Ciravegna, L. (2014). Differentiation strategies in emerging markets: The case of Latin American agribusinesses. En: Journal of Business Research, 67 (5): 847-855.

Bright, J.; Davies, R.E.; Downes C.A. \& Sweeting, R.C. (1992). The deployment of costing techniques and practices: a UK study. En: Management Accounting Research, 3 (3): 201-211.

Burkert, M.; Davila, A.; Mehta, K. \& Oyon, D. (2104). Relating alternative forms of contingency fit to the appropriate methods to test them. En: Management Accounting Research, 25 (1): 6-29.

Burns, T. \& Stalker, G.M. (1961). The management of innovation. Tavistock, London.

Chapman, C.S. (1998). Accountants in organizational networks. En: Accounting, Organizations and Society, 23 (8): 737-766.

Chenhall, R.; Hall, M. \& Smith, D. (2013). Performance measurement, modes of evaluation and the development of compromising accounts. En: Accounting, Organizations and Society, 38 (4): 268-287.

Chenhall, R.H. (2003). Management control systems design within its organizational context: finding from contingency-based research and directions for the future. En: Accounting, Organizations and Society, 28 (2-3): 127-168.

Chenhall, R.H. \& Langfield-Smith, K. (1998a). Adoption and benefits of management accounting practices: an Australian study. En: Management Accounting Research, 9 (1): 1- 19.
Chenhall, R.H. \& Langfield-Smith, K. (1998b). The relationship between strategies priorities, management techniques and management accounting: an empirical investigation using a systems approach. En: Accounting, Organizations and Society, 23 (3): 243-264.

Chenhall, R.H. \& Morris, D. (1986). The impact of Structure, Environment, and Interdependence on the Perceived Usefulness of Management Accounting Systems. En: The Accounting Review, 61: 16-35.

Chin, W.W. (1998). The Partial Least Squares Approach to Structural Equation Modeling, en G.A. Marcoulides [ed.]: Modern Methods for Business Research, 295-336. Mahwah, NJ: Lawrence Erlbaum Associates, Publisher.

Chong, V.K. \& Chong, K.M. (1997). Strategic choices, environmental uncertainty and SBU performance: a note on the intervening role of management accounting systems. En: Accounting and Business Research, 27 (4): 268-276.

Christensen, J. \& Demski, J. (2003). Accounting Theory-An Information Content Perspective. New York: McGraw-Hill.

Claver-Cortés, E.; Molina-Azorín, J.F. \& Pereira-Moliner, J. (2007). Competitiveness in mass tourism. En: Annals of Tourism Research, 34: 727-745.

Cooper, R. (1996). Costing techniques to support corporate strategy: evidence from Japan. En: Management Accounting Research, 7 (2): 219-246.

Cuadrado-Roura, J.R \& Maroto-Sánchez, A. (2012). Análisis del proceso de especialización regional en servicios en España. En: Eure, 33: 5-34.

Cugueró-Escofet, N. \& Rosanas, J.M. (2013). The just design and use of management control systems as requirements for goal congruence. En: Management Accounting Research, 24 (1): 23-40.

Donaldson, L. (2001). The contingency theory of organizations (Ed. Sage, California).

Drazin, R. \& Van de Ven, A.H. (1985). Alternative forms of fit in Contingency Theory. En: Administrative Science Quarterly, 30: 514-539.

Emmanuel, C.; Otley, D. \& Merchant, K. (1991). Accounting for Management Control. Londres Chapman\&Hall. Segunda edición impresa.

Fullerton, R.R. \& McWatters, C.S. (2002). The role of performance measures in relation to the degree of JIT implementation. En: Accounting, Organizations and Society, 27 (8): 711-735.

Fuchs, P.H.; Mifflin, K.E.; Miller, D. \& Whitney, J.O. (2000). Strategic integration: competing in the age of capabilities. En: California Management Review, Spring: 118-147.

Garrigos-Simon, F.J. \& Narangajavana, Y. (2005). Competitive strategies and performance in Spanish hospitality firms. En: International Journal of Contemporary Hospitality Management, 17: 22-38.

Gefen, D.; Straub, D.W. \& Boudreau, M.C. (2000). Structural equation modelling and regression: guidelines for research practice. En: Association for Information Systems, 4 (7): 1-70.

Gerdin, J. (2005). Management accounting system design in manufacturing departments: an empirical investigation using a multiple contingencies approach. En: Accounting, Organizations Society, 30 (2): 99-126.

González-Benito, J. (2010). Supply strategy and business performance: An analysis based on the relative importance assigned to generic competitive objectives. En: International Journal of Operations \& Production Management, 30 (8): 774-797. 
Gordon, L.A. \& Miller, D. (1976). A contingency framework for the design of accounting information systems. En: Accounting, Organizations and Society, 1 (1): 59-69.

Govindarajan, V. (1984). Appropriateness of accounting data in performance evaluation: and empirical examination of environmental uncertainty as an intervening variable. En: Accounting, Organizations and Society, 9 (2): 125-136.

Green, K.; Covin, J. \& Slevin, D. (2008). Exploring the relationship between strategic reactiveness and entrepreneurial orientation: The role of structure-style fit. En: Journal of Business Venturing, 23 (3): 356-383.

Guilding, C.; Carvens, K.S. \& Tayles, M. (2000). An international comparison of strategic management accounting practices. En: Management Accounting Research, 11 (1): 113-135.

Henri, J.F. (2006). Organizational culture and performance measurement systems. En: Accounting, Organizations and Society, 31: 77-103.

Hodge, B.J.; Anthony, W.P. \& Gales, L.M. (2003). Teoría de la organización. Un enfoque estratégico. Pearson. Prentice Hall, $6^{\circ}$ Ed.

Hunt, S.D. \& Morgan, R.M. (1995). The Comparative Advantage Theory of Competition. En: Journal of Marketing, 59 (April): 1-15.

IET Instituto de Estudios Turísticos (2012). Disponible: http://www. iet.tourspain.es/es-ES/Paginas/default.aspx.

Jermias, J. \& Gani, L. (2004). Integrating business strategy, organizational configurations and management accounting systems with business unit effectiveness: a fitness landscape approach. En: Management Accounting Research, 15 (2): 179-200.

Johnson, H.T. \& Kaplan, R.S. (1987). Relevance lost the rise and fall of management accounting. Boston MA: Harvard Business School Press.

Jorgensen, B. \& Messner, M. (2009). Management control in new product development: the dynamics of managing flexibility and efficiency. En: Journal of Management Accounting Research, 21 (1): 99-124.

Kajüter, P. \& Kulmala, H.I. (2005). Open-book accounting in networks potential achievements and reasons for failures. En: Management Accounting Research, 16 (2): 179-204.

Kanter, R. (2001). Evolve! Succeeding in the Digital Culture of Tomorrow. Harvard Business School Press, Boston.

Kaplan, R.S. (1984). Yesterday's accounting undermines production. En: Harvard Business Review, July-August: 95-101.

Keidel, R.W. (1994). Rethinking organizational design. En: Academy of Management Executive, 8 (4):12-17.

Khandwalla, P. (1977). Design of organizations, New York, Harcourt Brace Jovanovich.

Koen, C. (2004). The dialectics of globalizations: what are the effects for management and organization in Germany and Japan? En: Research in International Business and Finances, 18: 173-197.

Laitinen, E.K. (2014). Influence of cost accounting change on performance of manufacturing firms. En: Advances in Accounting, 30 (1): 230-240.

Langfield-Smith, K. (1997). Management control systems and strategy: a critical review. En: Accounting, Organizations and Society, 22 (2): 207-232.

Lawrence, P.R. \& Lorsch, J.W. (1967). Organizations and environment. Boston. M.A. Harvard Business School Press.

Macintosh, N. (1994). Management accounting and control systems: an organizational and behavioral approach. Chichester, UK: John Wiley.

Mahlendorf, M.D.; Kleinschmit, F. \& Perego, P. (2014). Relational effects of relative performance information: The role of pro- fessional identity. En: Accounting, Organizations and Society, 39 (5): 331-347.

Mauri, A.G. (2012). Hotel Revenue Management: Principles and Practices. Pearson Italia, Milano-Torino.

McManus, L. (2013).Customer accounting and marketing performance measures in the hotel industry: Evidence from Australia. En: International Journal of Hospitality Management, 33: 140-152.

Miller, D. (1988). Relating Porter's business strategies to environment and structure: analysis and performance implications. En: Academy of Management Journal, 31(2): 280-308.

Mundy, J. (2010). Creating dynamic tensions through a balanced use of management control systems. En: Accounting, Organizations and Society, 35 (5): 499-523.

Naranjo-Gil, D. \& Álvarez-Dardet, C. (2006). El uso del sistema contable de gestión en la implantación de la estrategia: análisis del ajuste contingente. En: Revista Española de Financiación y Contabilidad, 128: 157-179.

Olaru, M.; Maier, D.; Nicoarã, D. \& Maier, A. (2014). Establishing the basis for Development of an Organization by Adopting the Integrated Management Systems: Comparative Study of Various Models and Concepts of Integration. En: Procedia - Social and Behavioral Sciences, 109: 693-697.

Parthasarthy, R. \& Sethi, S.P. (1993). Relating strategy and structure to flexible automation: a test of fit and performance implications. En: Strategic Management Journal, 14: 529-549.

Perera, S.; Harrison, G. \& Poole, M. (1997). Customer-focused manufacturing strategy and the use of operations-based nonfinancial performance measures: a research note. En: Accounting, Organizations and Society, 22 (6): 557-572.

Podsakoff, P.M. \& Organ D.W. (1986). Common method biases in behavioral research: a critical review of the literature and recommended remedies. En: Journal of Management, 12: 531-544.

Porter, M.E. (1985). Competitive Advantage: Creating and Sustaining Superior Performance. New York: Free Press.

Puolamäki, E. (2004). Strategic Management Accounting. Constructions in Organizations. Turun Kauppakorkekoulun Julkaisuja. Turku School of Economics and Business Administration Publications.

Rigby, D.K. (2003). Management tools survey 2003; usage up as companies strive to make headway in tough times. En: Strategy \& Leadership, 31 (5): 4 -12.

Rigby, D.K. \& Bilodeau, B. (2013). Management Tools \& Trend 2013. http://bain.com/Images/BAIN_BRIEF_Management_ Tools \%26 Trends 2013.pdf.

Ripoll, V. \& Urquidi, A.C. (2010). Herramientas de contabilidad de gestión utilizadas en la práctica empresarial: una revisión crítica de los trabajos de investigación. En: Academia. Revista Latinoamericana de administración, 44:1-20.

Sanchez Quiros, I. (2003). Estrategia, estructura y cultura como factores clave de éxito en los hoteles españoles. IX Foro de Finanzas, ACEDE. Navarra.

Shields, M.D.; Kato, Y. \& Deng, J. (2000). The design of control systems: test of direct and indirect - effect models. En: Accounting, Organizations and Society, 25 (2): 185-202.

Sim, K.L. \& Killough, L.N. (1998). The performance effects of complementarities between manufacturing practices and management accounting systems. En: Journal of Management Accounting Research, 10: 325-346.

Sinkula, J.M.; Baker, W. \& Noordewier, T.G. (1997). A Framework for Market-Based Organizational Learning: Linking Values, 
Knowledge and Behaviour. En: Journal of the Academy of Marketing Science, 25 (Fall): 305-318.

Siti-Nabiha, A.K. \& Scapens, R.W. (2005). Stability and change: an institutionalism study of management accounting change. En: Accounting, Auditing \& Accountability Journal, 18 (1): 44-73.

Smith, J.; Yuhchang, H.; Pei, B. \& Reneau, J. (2002). The performance effects of congruence between product competitive strategies and purchasing management design. En: Management Science, 48 (7): 866-885.

Thomas, A.S. \& Ramaswamy, K. (1996). Matching managers to strategy: Further Tests of the Miles and Snow Typology. En: British Journal of Management, 7: 247-261.

Tuomimem, M. \& Möller, K. (1996). Market Orientation: A State of the Art Review, 25th EMAC Annual Conference, Marketing for an Expanding Europe, J. Beracs, A. Baner \& J. Simon. (eds.), Budapest, May. Proceedings, 2: 1161-1181.

Van der Voet, J. (2014). The effectiveness and specificity of change management in a public organization: Transformational leadership and a bureaucratic organizational structure. En: European Management Journal, 32, (3): 373-382.
VII Foro de Liderazgo Turístico de Exceltur (2014). El Turismo: Sector clave para reforzar la marca País. 21 de Enero. Madrid. Centro de Convenciones IFEMA.

Weißenberger, B \& Angelkort, H. (2011). Integration of financial and management accounting systems: The mediating influence of a consistent financial language on controllership effectiveness. En: Management Accounting Research, 22 (3): 160-180.

Werbel, J. \& DeMarie, S. (2005). Aligning strategic human resource management and person-environment fit. En: Human Resource Management Review, 15 (4): 247-262.

Ylinen, M. \& Gullkvist, B. (2014). The effects of organic and mechanistic control in exploratory and exploitative innovations. En: Management Accounting Research, 25 (1): 93-112.

Zhanga, D.; Lindermanb, K. \& Schroederc, R.G. (2012). The moderating role of contextual factors on quality management practices. En: Journal of Operations Management, 30 (1-2): 12-23.

Zhenga, W.; Yangb, B. \& McLeanc, G.N. (2010). Linking organizational culture, structure, strategy, and organizational effectiveness: Mediating role of knowledge management. En: Journal of Business Research, 63 (7): 763-77. 